\title{
Acute Ischemic Stroke Endovascular Treatment: Predictors for favourable Functional Outcome.
}

Authors: J. Carneado-Ruiz, Erika María Beck Román, Lorena Fernández Gil, Andrea Castro-Villacañas Farzamnia, L. Mena Romo, L. Alba Alcántara, R. Velasco Calvo, A. Ruiz Molina., R. González Santiago, P. Ruiz Garcia, A. Vega Astudillo,

Stroke Unit. Neurology Department. Hospital Universitario Puerta de Hierro, Majadahonda, Madrid (HUPH).

\section{OBJECTIVE:}

To analyse factors related to 90 days favourable functional outcome in patients with acute ischemic stroke treated with endovascular treatment.

\section{PATIENTS/METHODS:}

Observational prospective study. The period studied was from october 2013 to february 2017. Variables related to 90 days functional outcome (Rankin 0-2 vs 3-6) were analysed.

RESULTS: Number of patients 141, Age median (M) (intercuartil range IQ 1-3) : 68 ( 59-70) Female: $42 \%$, NIHSS basal $M(I Q$ 1-3): 19 (16-22), time from onset to arterial puncture: M(IQ 1-3): 264 (200-330), favourable arterial recanalization (TICI 3/2b): $70,21 \%$. Rankin0-2 90 days: $46.8 \%$

Differences between groups (Rankin 0-2 vs 3-6 90 days) were analysed.

A univariate analysis is performed and a multivariate predictor model is developed : variables selected in the model were : basal glucemia OR $(\mathrm{Cl} 95 \%)$ 0,98 (0.97-0.99) $\mathrm{p}=0.038$; basal NIHSS OR ( $\mathrm{Cl} 95 \%$ ) 0,87 ( 0.79-0.95) p=0.004, time from onset to arterial recanalization OR $(\mathrm{Cl} 95 \%) \quad 0,996$ (0.993- 1.00) $\mathrm{p}=0.061$, favourable arterial recanalization $(\mathrm{TICl} 2 \mathrm{~B} / 3)$ OR $(\mathrm{Cl}$ $95 \%$ ) $14.04(4.38-44.9) \mathrm{p}<0.001$ and presence of hemorragic transformation: parenchymatous hemorraghe type $(\mathrm{PH}) 0.20$ (0.04-0.91)

The ROC Curve for the model demonstrated an area under the curve: 0.83 for predicting Rankin 0-2 at 90 days

\begin{tabular}{|l|l|}
\hline Patient Characteristics & \\
\hline Number & 141 \\
\hline Age Median (IQR 1-3) & $68(78-59)$ \\
\hline Gender Male N (\%) & $82(58 \%)$ \\
\hline SBP mmHg median (IQR 1-3) & $144(158-126)$ \\
\hline DBP mmHg median (IQR 1-3) & $80(90-70)$ \\
\hline FRVn median (IQR 1-3) & $3(2-4)$ \\
\hline Glucose mg/dl & $126(152-106)$ \\
\hline NIHSS basal median (IQR 1-3) & $19(22-16)$ \\
\hline NIHSS 24h median (IQR 1-3) & $9(18-2)$ \\
\hline Rankin 0-2 3 months N (\%) & $66(46,8 \%)$ \\
\hline TH total N (\%) & $45(31,9 \%)$ \\
\hline TH symptomatic N (\%) & $11(7,8 \%)$ \\
\hline TH PH2 N (\%) & $18(12,7 \%)$ \\
\hline Combined treatment (IV/IA) N (\%) & $72(51,1 \%)$ \\
\hline Recanalization TICI2b-3 N (\%) & $99(70,2 \%)$ \\
\hline Time ER-IA (min) median (IQR 1-3) & $141(89-204)$ \\
\hline Time to IA (min.) median (IQR 1-3) & $264(330-200)$ \\
\hline Time to Recanalization (min.) median & $350(282-426)$ \\
\hline (IQR 1-3) & $31(22,1 \%)$ \\
\hline Tandem Oclusion N (\%) & \\
\hline
\end{tabular}

Rha JH, Saver JL. The impact of recanalization on ischemic stroke outcome: A meta-analysis. Stroke. 2007;38:967 ..-73. Lees KR, Bluhmki E, von Kummer R, et al. Time to treatment with intravenous alteplase and outcome in stroke: an updated pooled analysis of ECASS, ATLANTIS, NINDS, and EPITHET trials. Lancet 2010; $375: 1695$
CONCLUSIONS: Predictors for favourable 90 days outcome in patients with acute ischemic stroke treated with endovascular treatment were : in a negative sense basal glucemia, basal NIHSS, time from onset to arterial recanalization and $\mathrm{PH}$; in a positive sense favourable arterial recanalization ( $\mathrm{TICl} 2 \mathrm{~B} / 3$ ) being the most relevant factor OR (Cl 95\% ) $14.04(4.38-44.9) \mathrm{p}$ $<0.001$.

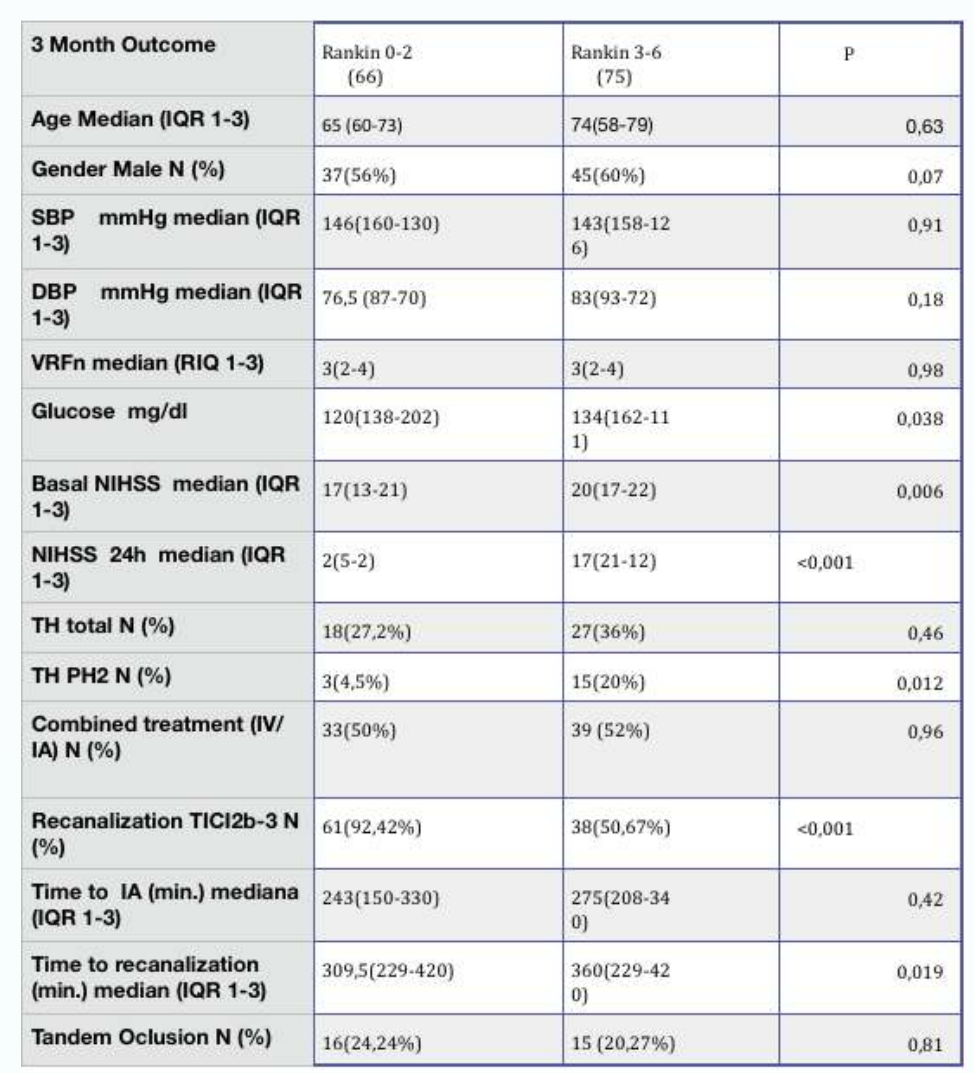

MULTIVARIATE ANALYSIS FOR FUNCTIONAL OUTCOME (RANKIN 0-2) AT 3 MONTHS.

\begin{tabular}{|l|l|l|}
\hline & OR (95\% IC) & P \\
\hline Basal NIHSS & $0,87(0.79-0.95)$ & 0,004 \\
\hline Basal Glucemia & $0,98(0.97-0.99)$ & 0,038 \\
\hline Time to recanalization $(\mathrm{min})$ & $0,996(0.993-1.00)$ & 0,061 \\
\hline TICI2b-3 & $14.04(4.38-44.9)$ & $<0,001$ \\
\hline Hemorrhage PH2 & $0.20(0.04-0.91)$ & 0,038 \\
\hline
\end{tabular}

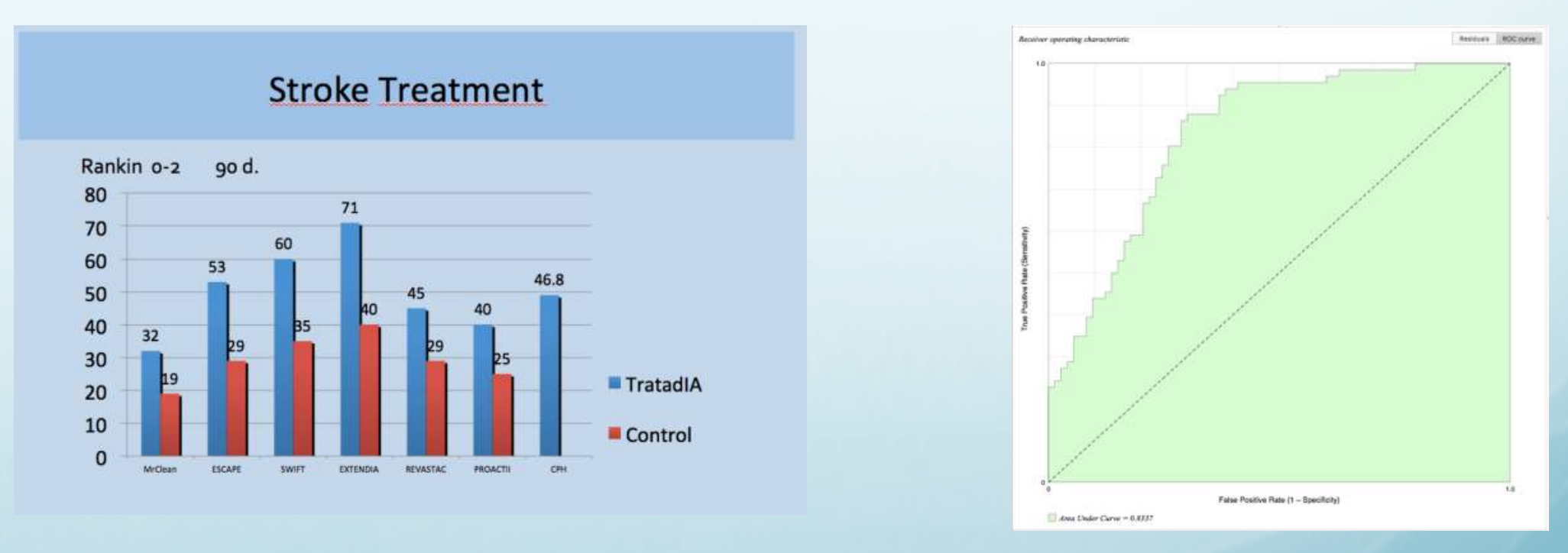

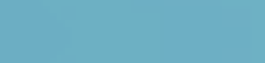

